\title{
Performance Analysis of MRC Receivers with Adaptive Modulation and Coding in Rayleigh Fading Correlated Channels with Imperfect CSIT
}

\author{
Ramiro Sámano-Robles, ${ }^{1}$ Egons Lavendelis, ${ }^{2}$ and Eduardo Tovar ${ }^{1}$ \\ ${ }^{1}$ Research Centre in Real-Time and Embedded Computing Systems, Porto, Portugal \\ ${ }^{2}$ Department of System Theory and Design, Riga Technical University, Riga, Latvia \\ Correspondence should be addressed to Ramiro Sámano-Robles; rasro@isep.ipp.pt
}

Received 19 July 2016; Revised 6 October 2016; Accepted 7 November 2016; Published 15 January 2017

Academic Editor: Stefano Savazzi

Copyright ( 2017 Ramiro Sámano-Robles et al. This is an open access article distributed under the Creative Commons Attribution License, which permits unrestricted use, distribution, and reproduction in any medium, provided the original work is properly cited.

\begin{abstract}
This paper addresses the performance analysis of an adaptive wireless link with one antenna transmitter and a multiple antenna maximum-ratio combining (MRC) receiver. Two main assumptions are used in this paper: (1) Rayleigh fading correlated channels (i.e., MRC branch correlation) and (2) imperfect (outdated) channel state information at the transmitter (CSIT) side. The main contribution of this work lies in the derivation of analytic expressions (in terms of a series expansion) of the statistics of correct packet reception conditional on the decisions made by the transmitter based on outdated CSIT. The novelty of this derivation is the joint modelling of spatially correlated branches, imperfect CSIT, and adaptive modulation based on threshold-trigger decision. Contrary to common belief, the results presented here suggest that spatial correlation not always affects the performance of the MRC receiver: at low signal-to-noise ratio (SNR), correlation can improve performance rather than degrading it. In contrast, at high SNR, correlation is found to always degrade performance. At high SNR, correlation tends to worse the degrading effects of imperfect CSIT, particularly when the number of antennas increases. Imperfect CSIT causes errors in the assignment of MCSs, thus reducing throughput performance. These errors become more evident in the high SNR regime, particularly when the values of branch correlation and the number of antennas increase.
\end{abstract}

\section{Introduction}

Wireless networks are rapidly evolving. New processing tools are constantly developed to cope more efficiently with impairments such as interference and fading. In addition, application layers are becoming increasingly complex. It has been proved that the conventional layered design is inadequate to cope with these new processing and application demands [1-4].

In cross-layer design, layers are now able to exchange information between them or in some cases they can even be fully merged or codesigned [3]. Cross-layer design is key for networks with multiple antennas and multiple interfering links [2-6], where layers can become tightly coupled.

A common issue in cross-layer design is optimization complexity. All the details of lower layers cannot be practically used in upper-layer optimization due to complexity constraints. Interface or abstraction models are thus required that provide a fair, but flexible representation of lower layers to be used in upper-layer optimization.

Another issue in cross-layer design is the passing of imperfect signalling information across layers. Cross-layer design increases the amount of signalling or bandwidth of feedback channels. Since feedback resources are limited and/or increasingly imperfect with higher bandwidths, signalling is subject to errors and/or outdated measurements.

This paper deals with the link-layer modelling in Rayleigh fading correlated channels of an adaptive wireless link using maximum-ratio combining (MRC) receiver and limited (outdated) feedback. Link-layer throughput is evaluated by means of an interface model based on an adaptive SNR (signalto-noise ratio) switching threshold scheme for modulation assignment. This model aims to provide an accurate but flexible representation of the physical (PHY) layer suitable 
for upper-layer design. In the proposed model, a packet transmission using a given modulation and coding scheme (MCS) is considered as correctly received with particular values of block-error rate (BLER) and spectral efficiency whenever the instantaneous SNR exceeds the reception threshold of the selected MCS. The reception parameters of each MCS are obtained from look-up-tables (LUTs) previously calculated via off-line PHY-layer simulation. The transmitter selects the most adequate MCS based on an estimated CSI. This CSI is assumed to have been initially collected by the receiver (perfect estimation) and subsequently reported back to the transmitter via a feedback channel affected by delay. The paper presents the analysis of the statistics of correct reception process conditional on the decision made by the transmitter (MCS selection) based on the inaccurate CSIT. The main contribution of this work is the joint analysis of spatial correlation and imperfect CSIT in link adaptation for multiple antenna MRC receivers.

This paper is organized as follows. Section 2 describes previous works and the achievements of this paper with respect to the state of the art. Section 3 describes the system model and the assumptions of the paper. Section 4 presents the link-layer interface model. Section 5 presents analytic results and sketches of the statistics of packet reception. Finally, Section 6 presents the conclusions of the paper.

\section{Previous Works and Paper Contributions}

Abstraction or interface models are relevant in the design of wireless random access assisted by signal processing. In these modern decentralized systems, the conventional collision model (where PHY-layer information is ignored) is no longer useful [1], as reception now depends on two aspects: PHY-layer and load conditions. Protocol design requires a performance model with enriched but compressed PHYlayer information. A reception model based on switching thresholds of signal-to-interference-plus-noise ratio (SINR) has been used in [7] for the study of a multihop ALOHA. The work in [8] proposed a stochastic reception matrix that introduces multipacket reception (the ability of the PHYlayer to correctly decode concurrent transmissions) in the optimization of ALOHA. The extension to the asymmetrical case was achieved in [9] using a conditional reception probability space.

Interface models are also used in broadband wireless networks with spatial diversity (see [10-12]). A summary of methodologies for link-to-system level interface (LSLI) modelling of broadband wireless networks can be found in [13]. Three main stages have been identified for LSLI: extraction of metrics, compression, and mapping. The proposed model in this paper contains instances of these three stages for the representation of adaptive MRC systems in resource allocation problems. LSLI models for multiple-input multiple-output (MIMO) systems were proposed in [14, 15]. An exponential function model of BLER has been proposed for the design of adaptive MIMO systems in [16]. Interface models are often required when some of the system processes are too complex to be included in detail within the main simulation loop or a higher-level design stage.

The simplest multiple antenna system is the MRC receiver, which provides a relatively flexible framework for statistical analysis and interface modelling. The literature of MRC receivers has focused on the derivation of outage and bit error probability distributions (see [17-25]). The effects of imperfect channel knowledge at the receiving end on the performance of MRC in Rayleigh fading correlated channels can be found in [17]. An extension to Rician channels was addressed in [18] following the analysis with perfect channel estimation presented in [19]. A series expansion of the statistics of MRC receivers with correlated Rician channels is given in [20]. A unified approach for analysis of two-stage MRC receivers with hybrid selection in generalized Rice correlated channels was proposed in [21]. Extensions to the case of cochannel interference are given in [22-25].

The present work considers the extension of outage probability analysis of MRC receivers to the study of adaptive modulation and coding (AMC) in Rayleigh fading correlated channels with imperfect/outdated CSIT. To the best of our knowledge, this is the first attempt in the literature that addresses these issues simultaneously. This work attempts to extend the analysis of MRC receivers towards including resource allocation aspects which are typical of upper-layer design (radio resource management). In addition, network design and in particular resource allocation for multiple antenna systems are usually conducted under the assumption perfect CSIT. This work attempts to advance this analysis to include different levels of CSI quality at the transmitter side. Imperfect CSIT has been addressed in [26] for distributed systems and in [27] for energy efficient MIMO link adaptation. In comparison with these works, which are focused on numerical evaluation of imperfect CSIT, this work provides an analytic framework for obtaining the statistics of errors in MCS assignment for correlated MRC receivers. This paper is the extension of the conference papers in $[28,29]$.

A related topic is the analysis of the effects of limited feedback in adaptive modulation for beam-forming and multiple antenna systems. The work in [30] provides a review of the state of the art of limited feedback in adaptation schemes for MIMO systems. The work in [31] presents the analysis of adaptive modulation for two-antenna beam-formers considering mean CSI at the transmitter side. The work in [32] addressed the impact of outdated feedback on AMC and user selection diversity systems for MIMO systems in Rayleigh uncorrelated channels. Other works with limited feedback for different types of system can be found in [3335]. All of these previous works consider uncorrelated MIMO channels. This work goes beyond this assumption searching for a joint analysis of limited feedback and spatial correlation for adaptive MRC receivers.

The results show that channel correlation not always affects the performance of link-layer throughput. It is also observed that correlation can improve system performance when the operational SNR is relatively low in comparison with the target SNR of the selected MCS. This low SNR effect is shown to be less evident in the presence of imperfect CSIT and also when the number of antennas of the MRC receiver 


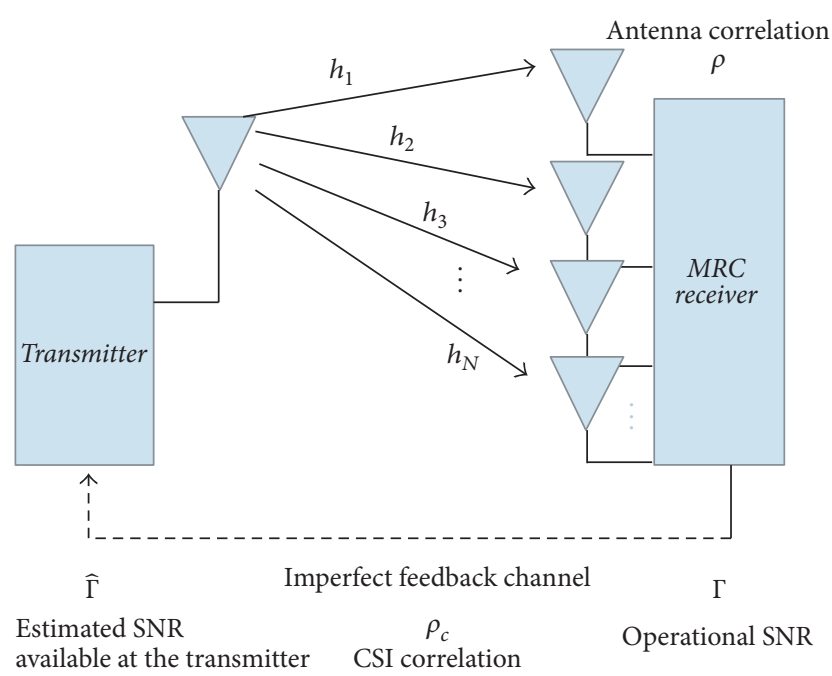

FIGURE 1: System model for an AMC-MRC receiver with imperfect CSIT.

increases. By contrast, at high SNR, channel correlation is shown to always affect performance of the MRC receiver. Details of the results follow in the main body of the paper.

\section{System Model}

Consider the wireless link depicted in Figure 1 with one transmitter and one receiver. The transmitter uses a single antenna while the receiver is enabled with a multiple antenna using maximum-ratio combining (MRC). The channel between the transmitter and the multiple antenna receiver is denoted by $\mathbf{h}=\left[h_{1}, h_{2}, \ldots, h_{N}\right]^{T}$, where $(\cdot)^{T}$ is the vector transpose operator and $N$ is the number of antennas. All channels will be modelled as zero-mean complex circular symmetrical Gaussian random variables with variance denoted by $\gamma: h_{n} \sim$ $\mathscr{C} \mathcal{N}(0, \gamma)$. In this paper it is assumed that the received signals of all antennas experience the same statistical correlation (assumption valid with low numbers of antennas or with small dimensions, where all antennas may experience similar signal statistics), which means that $E\left[h_{k}^{*} h_{n}\right]=\rho \gamma$, where $k \neq n,(\cdot)^{*}$ is the complex conjugate operator, $E[\cdot]$ is the statistical average operator, and $\rho$ is the spatial correlation coefficient.

The transmitted signal with $Q$ symbols is denoted by $\mathbf{w}=$ $[w(0), w(1), \ldots, w(Q-1)]^{T}$. This signal will use one of $M$ modulation and coding schemes (MCSs). These MCSs are arranged in increasing order according to their target signalto-noise ratio (SNR). The target SNR of the $m$ th MCS will be denoted by $\beta_{m}$. The variables $\theta_{m}$ and $\eta_{m}$ will denote, respectively, the BLER and spectral efficiency (in bps/Hz) values considering operation at the target SNR of the $m$ th MCS. It is assumed that the receiver monitors the quality of the channel and reports it back to the transmitter. Based on this collected channel state information (CSI), the transmitter selects the most appropriate MCS. This paper considers perfect channel estimation at the receiver side and imperfect channel state information at the transmitter side (CSIT).
Imperfect CSIT is assumed to be mainly due to a feedback channel affected by delay. All the estimated channel variables available at the transmitter side that will be used for MCS selection will be expressed using the following notation $\widehat{h}_{n}$. The estimated channel available at the transmitter will be thus denoted by $\widehat{\mathbf{h}}=\left[\widehat{h}_{1}, \widehat{h}_{2}, \ldots, \widehat{h}_{N}\right]^{T}$, where $E\left[\widehat{h}_{k}^{*} \widehat{h}_{n}\right]=\rho \gamma, k \neq n$.

The signal received by the $n$th antenna of the MRC receiver can be expressed as follows:

$$
\mathbf{r}_{n}=h_{n} \mathbf{w}+\mathbf{v}_{n},
$$

where $\mathbf{v}_{n}=[v(0), v(1), \ldots, v(Q-1)]^{T}$ is the vector of zero-mean complex circular Gaussian noise with covariance matrix $\mathbf{I} \sigma_{v}^{2}: \mathbf{v} \sim \mathscr{C} \mathscr{N}\left(\mathbf{0}_{\mathrm{Q}}, \sigma_{v}^{2} \mathbf{I}_{\mathrm{Q}}\right)$, where $\mathbf{0}_{\mathrm{Q}}$ and $\mathbf{I}_{\mathrm{Q}}$ are, respectively, the vector of zeros and the identity matrix of order $Q$, and $\sigma_{v}^{2}$ is the noise variance. Let us now consider that the transmitted symbol complies with the following power constraint: $E\left[\mathbf{w}^{H} \mathbf{w}\right]=P$, where $(\cdot)^{H}$ is the Hermitian vector transpose operator. The receiver uses MRC to process the signals received by all the antennas: $\mathbf{h}^{H} \mathbf{R}$, where $\mathbf{R}$ is the collected signals of all the antennas. This leads to the wellknown formula for the postprocessing or instantaneous signalto-noise ratio (SNR) of the MRC receiver considering perfect channel estimation at the receiver side [21]:

$$
\Gamma=\sum_{n=1}^{N} \frac{P\left|h_{n}\right|^{2}}{\sigma_{v}^{2}} .
$$

The SNR measured by the terminal is reported back to the transmitter via a feedback channel mainly affected by delay. The estimated (outdated) SNR available at the transmitter side will be expressed as follows:

$$
\widehat{\Gamma}=\sum_{n=1}^{N} \frac{P\left|\widehat{h}_{n}\right|^{2}}{\sigma_{v}^{2}} .
$$

For convenience, all estimated channels will be expressed using a linear correlation model similar to the one used in [21], which in our context will be written as follows:

$$
\widehat{h}_{n}=\sqrt{1-\rho} Z_{n}+\sqrt{\rho} G,
$$

where the variables $Z_{n}$ and $G$ are identically and independently distributed (i.i.d.) zero-mean complex circular symmetrical Gaussian random variables with variance $\gamma$ : $\left\{Z_{n}, G\right\} \sim \mathscr{C} \mathscr{N}(0, \gamma)$. Note that the previous correlation model complies with $E\left[\widehat{h}_{n}^{*} \widehat{h}_{\tilde{n}}\right]=\rho \gamma$ and $E\left[\widehat{h}_{n}^{*} \widehat{h}_{\tilde{n}}\right]=\gamma$.

To model the statistical correlation between the instantaneous and the estimated CSIT, let us propose another linear correlation model that complies with $E\left[h_{n}^{*} \widehat{h}_{n}\right]=\rho_{c} \gamma$ and $E\left[h_{n}^{*} h_{n}\right]=\gamma$. This model has been used in the literature of error quantization analysis for multiple antenna systems with adaptive modulation (see [30-36]). It is also the most used correlation model for two Gaussian random variables:

$$
h_{n}=\rho_{c} \widehat{h}_{n}+\sqrt{1-\rho_{c}^{2}} Y_{n}
$$

where $\rho_{c}$ is the temporal correlation coefficient between the instantaneous $\left(h_{n}\right)$ and the estimated CSI $\left(\widehat{h}_{n}\right)$ available at the 
transmitter side. This coefficient also describes the accuracy of the CSIT ( $\rho_{c}=1$ indicates perfect CSIT). All variables $Y_{n}$ in (5) are i.i.d. zero-mean complex circular symmetrical Gaussian random variables with variance $\gamma: Y_{n} \sim \mathscr{C} \mathscr{N}(0, \gamma)$. The correlation model in (5) is a simple modification of the linear correlation model used for MRC branch correlation modelling in (4). The correlation coefficient can be obtained by the well-known formula of the Jakes model for mobile systems $\rho_{c}=J_{0}\left(2 \pi f_{d} \delta_{t}\right)$, where $J_{0}$ is the modified Bessel function or order zero, $f_{d}$ is the maximum Doppler shift, and $\delta_{t}$ is the time difference between observations.

\section{Link Performance Model}

This section deals with the link performance modelling of the multiple antenna AMC-MRC receiver under the assumption of imperfect (outdated) CSIT. It is assumed that the $m$ th MCS is selected when the estimated SNR of the MRC receiver lies within the range $\beta_{m} \leq \widehat{\Gamma}<\beta_{m+1}$ (for practical purposes the upper threshold for the Mth MCS is considered to be set to a very large value: $\left.\beta_{M+1} \rightarrow \infty\right)$. Therefore, the probability that the $m$ th MCS is selected for transmission can be written as

$$
\operatorname{Pr}\left\{\beta_{m} \leq \widehat{\Gamma}<\beta_{m+1}\right\} .
$$

Link-layer throughput (denoted by $T$ ) will be expressed as a linear contribution of all possible MCSs with their respective selection and conditional reception probabilities weighted by their conditional throughput performance $\left(T_{m}\right)$ :

$$
\begin{aligned}
T= & \sum_{m=1}^{M} E_{\Gamma}\left[T_{m}(\Gamma) \mid \beta_{m} \leq \widehat{\Gamma}<\beta_{m+1}\right\} \\
& \cdot \operatorname{Pr}\left\{\beta_{m} \leq \widehat{\Gamma}<\beta_{m+1}\right\}
\end{aligned}
$$

where $T_{m}(\Gamma)$ indicates the link-layer throughput when using the $m$ th MCS conditional on a particular value of the operational SNR $\Gamma$. In this paper we consider a simplification of this expression by assuming that the term $T_{m}(\Gamma)$ is a step function defined by a switching SNR threshold $\left(\beta_{m}\right)$ above which all packet transmissions are assumed to be correctly received with a given $\operatorname{BLER}\left(\theta_{m}\right)$ and spectral efficiency $\left(\eta_{m}\right)$. The simplification can be expressed as follows:

$$
\begin{aligned}
T= & \sum_{m=1}^{M} \operatorname{Pr}\left\{\Gamma \geq \beta_{m} \mid \beta_{m} \leq \widehat{\Gamma}<\beta_{m+1}\right\} \\
& \times \Delta_{\mathrm{BW}} \eta_{m}\left(1-\theta_{m}\right) \operatorname{Pr}\left\{\beta_{m} \leq \widehat{\Gamma}<\beta_{m+1}\right\},
\end{aligned}
$$

where (i) $\Delta_{\mathrm{BW}}$ is the operational bandwidth in $\mathrm{Hz}$ and (ii) $\operatorname{Pr}\left\{\Gamma \geq \beta_{m} \mid \beta_{m} \leq \widehat{\Gamma}<\beta_{m+1}\right\}$ is the probability of the instantaneous SNR $\Gamma$ to surpass the threshold $\beta_{m}$ provided the estimated SNR $\widehat{\Gamma}$ (used for MCS selection) lies in the range $\left[\beta_{m}, \beta_{m+1}\right)$.

Note that this last conditional probability term captures the effects of imperfect CSIT on the performance of the adaptation scheme. In the case of perfect CSIT $\left(\rho_{c}=1\right)$, correct reception occurs with probability one. Also note that the link-layer throughput expression in (8) represents only an approximation (compression) of the real performance of the system. The simplified model in (8) assumes packets are erroneous when the instantaneous SNR drops below the reception threshold $\beta_{m}$, when in practice there might be some cases where correct reception can still occur. Conversely, some cases with higher instantaneous SNR than the reception threshold could also lead to erroneous packet transmissions. Accurate BLER curve fitting modelling has been proposed in [16] using exponential functions for $T_{m}$ in (7) considering different values of SNR that capture in more detail the performance of the PHY-layer. In comparison with this previous approach, the objective in this paper is to provide a flexible representation of the link-layer throughput that can used in upper-layer design enriched with PHY-layer information (compressed). This type of compression/abstraction model as in (8) has been proved accurate for system level simulation of networks with considerable excursions of path-loss values [12], which are typical of cellular systems where users lie at different distances from the access points. Link-layer throughput expression in (8) can be represented as a generic link performance model as described in [13] and illustrated in Figure 2. The quality metrics of the model are the SNR value of the MRC receiver, the correlation statistics, and the power settings. The compression stage is achieved by representing the MRC receiver with a unique spatial correlation coefficient and the switching threshold assumption in (8). The mapping stage is achieved by using look-up-tables (LUTs) for the values of spectral efficiency and BLER in expression (8) obtained over AWGN channels.

The following subsections present the derivation of analytic expressions of the different terms of the throughput model in (8). For convenience, it is useful to derive the statistics of the estimated SNR $\widehat{\Gamma}$ (presented in Section 4.1) and then deal with the statistics of the instantaneous SNR $\Gamma$ (presented in Section 4.2) conditional on the MCS selection process.

4.1. Statistics of $\widehat{\Gamma}$. Let us now substitute the correlation model described by (4) in the expression of the estimated SNR in (3):

$$
\widehat{\Gamma}=\sum_{n=1}^{N} \frac{P\left|\sqrt{1-\rho} Z_{n}+\sqrt{\rho} G\right|^{2}}{\sigma_{v}^{2}} .
$$

Consider now the previous expression conditional on an instance of the random variable $G$. Under this assumption, the expression in (9) becomes the summation of the squares of Gaussian complex variables $\sqrt{P(1-\rho) / \sigma_{v}^{2}} Z_{n}$ each one with a mean given by $\sqrt{P \rho / \sigma_{v}^{2}} G$. Therefore, the estimated SNR of the MRC receiver $\widehat{\Gamma}$ conditional on an instance of random variable $G$ has a noncentral chi-square distribution with $2 \mathrm{~N}$ degrees of freedom. The conditional characteristic function (CF) of $\widehat{\Gamma}$ can be thus written as (see [37] for details of chisquare distributions)

$$
\Psi_{\widehat{\Gamma} \mid G}(i \omega)=(1-i \omega \widetilde{\gamma})^{-N} e^{i \omega \alpha|G|^{2} /(1-i \omega \tilde{\gamma})},
$$

where $\tilde{\gamma}=P(1-\rho) \gamma / \sigma_{v}^{2}, i=\sqrt{-1}, \omega$ is the frequency domain variable, $\alpha=P N \rho / \sigma_{v}^{2}$, and $\Psi_{x \mid y}$ denotes the CF of random 


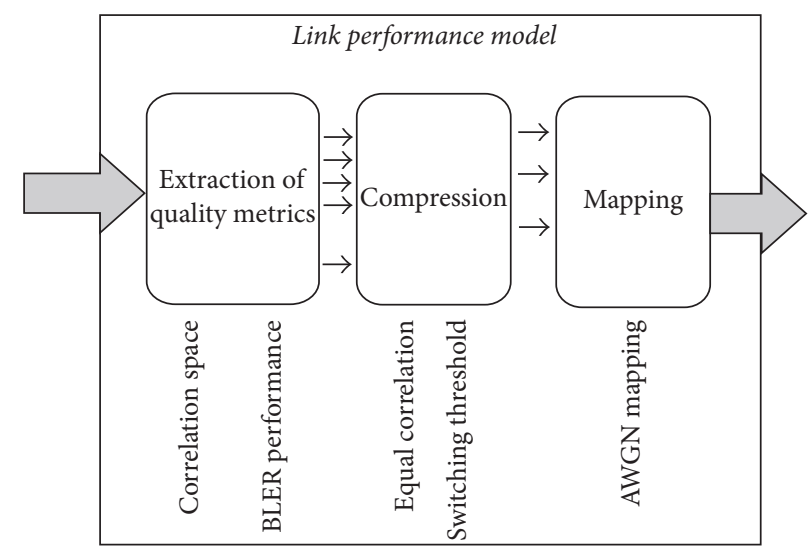

FIGURE 2: Stages of link performance model for cross-layer resource allocation [13].

variable $X$ conditional on an instance of random variable $Y$, for any $X$ and $Y$ random variables. By using the following change of variable $x=|G|^{2}$, the expression in (10) becomes

$$
\Psi_{\widehat{\Gamma} \mid x}(i \omega)=(1-i \omega \widetilde{\gamma})^{-N} e^{i \omega \alpha x /(1-i \omega \widetilde{\gamma})} .
$$

The unconditional CF of the estimated SNR can be now obtained by averaging the previous expression over the probability density function (PDF) of $x=|G|^{2}$, which under the Rayleigh fading assumption is given by $f_{x}(x)=$ $(1 / \gamma) e^{-x / \gamma}$. Therefore, the unconditional CF of the estimated SNR can be obtained as follows:

$$
\Psi_{\widehat{\Gamma}}(i \omega)=\int_{0}^{\infty}(1-i \omega \widetilde{\gamma})^{-N} e^{i \omega \alpha x /(1-i \omega \widetilde{\gamma})} \gamma^{-1} e^{-x / \gamma} d x
$$

which after the integration (see Appendix A) becomes

$$
\Psi_{\widehat{\Gamma}}(i \omega)=(1-i \omega \breve{\gamma})^{-1}(1-i \omega \widetilde{\gamma})^{1-N}
$$

where $\breve{\gamma}=\alpha \gamma+\widetilde{\gamma}$. The expression in (13) can be rewritten using partial fraction expansion (PFE):

$$
\Psi_{\widehat{\Gamma}}(i \omega)=A(1-i \omega \breve{\gamma})^{-1}+\sum_{n=1}^{N-1} B_{n}(1-i \omega \widetilde{\gamma})^{-n}
$$

where $A=(1-\tilde{\gamma} / \breve{\gamma})^{1-N}$ and $B_{n}=(-\tilde{\gamma} / \breve{\gamma})(1-\tilde{\gamma} / \breve{\gamma})^{n-N}$. For details of this derivation see Appendix B. The back-transform of (14) yields a PDF and complementary cumulative distribution function (CCDF) given, respectively, by

$$
\begin{aligned}
& f_{\widehat{\Gamma}}(y)=\frac{A}{\breve{\gamma}} e^{-y / \breve{\gamma}}+e^{-y / \tilde{\gamma}} \sum_{n=1}^{N-1} \frac{B_{n} y^{n-1}}{\widetilde{\gamma}^{n}(n-1) !}, \\
& \bar{F}_{\widehat{\Gamma}}(y)=A e^{-y / \breve{\gamma}}+e^{-y / \tilde{\gamma}} \sum_{n=1}^{N-1} \sum_{u=0}^{n-1} \frac{B_{n} y^{u}}{\widetilde{\gamma}^{u} u !} .
\end{aligned}
$$

Using this result, the probability of selecting the $m$ th MCS in (6) can be written as

$$
\operatorname{Pr}\left\{\beta_{m} \leq \widehat{\Gamma}<\beta_{m+1}\right\}=\bar{F}_{\widehat{\Gamma}}\left(\beta_{m}\right)-\bar{F}_{\widehat{\Gamma}}\left(\beta_{m+1}\right) .
$$

4.2. Statistics of $\Gamma$. This subsection deals with the statistics of the instantaneous SNR $\Gamma$ considering the statistics of the estimated SNR $\widehat{\Gamma}$ obtained in the previous subsection. Let us first substitute the correlation model described by (5) in the expression of the instantaneous SNR of the MRC receiver in (2):

$$
\Gamma=\sum_{n=1}^{N} \frac{P\left|\rho_{c} \widehat{h}_{n}+\sqrt{1-\rho_{c}^{2}} Y_{n}\right|^{2}}{\sigma_{v}^{2}} .
$$

Consider now the previous expression conditional on an instance of the random variables $\widehat{h}_{n}$. Under this assumption, the expression in (9) becomes the summation of the squares of Gaussian complex variables $\sqrt{P\left(1-\rho_{c}^{2}\right) / \sigma_{v}^{2}} Y_{n}$ each one with mean $\sqrt{P / \sigma_{v}^{2}} \rho_{c} \widehat{h}_{n}$. The instantaneous SNR $\Gamma$ of the MRC receiver conditional on $\widehat{h}_{n}$ has a noncentral chi-square distribution with $2 N$ degrees of freedom. The conditional CF is thus given by

$$
\Psi_{\Gamma \mid \widehat{h}_{n}}(i \omega)=(1-i \omega \bar{\gamma})^{-N} e^{i \omega \rho_{c}^{2} \sum_{n=1}^{N}\left(P\left|\widehat{h}_{n}\right|^{2} / \sigma_{v}^{2}\right) /(1-i \omega \bar{\gamma})},
$$

where $\bar{\gamma}=P\left(1-\rho_{c}^{2}\right) \gamma / \sigma_{v}^{2}$. By using the following change of variable $\widehat{\Gamma}=\sum_{n=1}^{N}\left(P\left|\widehat{h}_{n}\right|^{2} / \sigma_{v}^{2}\right)$, the expression in (19) becomes

$$
\Psi_{\Gamma \mid \widehat{\Gamma}}(i \omega)=(1-i \omega \bar{\gamma})^{-N} e^{i \omega \rho_{c}^{2} \widehat{\Gamma} /(1-i \omega \bar{\gamma})} .
$$

The CF of the instantaneous SNR conditional on the estimated SNR being in the range $\beta_{m} \leq \widehat{\Gamma}<\beta_{m+1}$ can be obtained by averaging the previous expression over the PDF of $\widehat{\Gamma}$ in (15). This leads to

$$
\begin{aligned}
& \Psi_{\Gamma \mid \beta_{m} \leq \widehat{\Gamma}<\beta_{m+1}}(i \omega) \\
& \quad=\int_{\beta_{m}}^{\beta_{m+1}}(1-i \omega \bar{\gamma})^{-N} e^{i \omega \rho_{c}^{2} \widehat{\Gamma} /(1-i \omega \bar{\gamma})} f_{\widehat{\Gamma}}(\widehat{\Gamma}) d \widehat{\Gamma},
\end{aligned}
$$




$$
\begin{aligned}
\Psi_{\Gamma \mid \beta_{m} \leq \widehat{\Gamma}<\beta_{m+1}}(i \omega)= & \frac{A\left(e^{-\beta_{m}(1-i \omega \dot{\gamma}) / \breve{\gamma}(1-i \omega \bar{\gamma})}-e^{-\beta_{m+1}(1-i \omega \dot{\gamma}) / \breve{\gamma}(1-i \omega \bar{\gamma})}\right)}{(1-i \omega \dot{\gamma})(1-i \omega \bar{\gamma})^{N-1}} \\
& +\sum_{n=1}^{N-1} \sum_{k=0}^{n-1} \frac{B_{n}\left(\beta_{m}^{k} e^{-\beta_{m}(1-i \omega \ddot{\gamma}) / \tilde{\gamma}(1-i \omega \bar{\gamma})}-\beta_{m+1}^{k} e^{-\beta_{m+1}(1-i \omega \ddot{\gamma}) / \widetilde{\gamma}(1-i \omega \bar{\gamma})}\right)}{k ! \tilde{\gamma}^{k}(1-i \omega \ddot{\gamma})^{n-k}(1-i \omega \bar{\gamma})^{N-n+k}},
\end{aligned}
$$

where $\dot{\gamma}=\rho_{c}^{2} \breve{\gamma}+\bar{\gamma}$ and $\ddot{\gamma}=\rho_{c}^{2} \widetilde{\gamma}+\bar{\gamma}$. The back-transform of the expression in (22) can be proved (see Appendix D) to result in the following CCDF for $\Gamma$ conditional on $\beta_{m} \leq \widehat{\Gamma}<\beta_{m+1}$ :

$$
\begin{array}{r}
\bar{F}_{\Gamma \mid \beta_{m} \leq \widehat{\Gamma}<\beta_{m+1}}(y)=A\left[\bar{\Xi}\left(-\frac{\beta_{m}}{\breve{\gamma}}, \dot{\gamma}, \bar{\gamma}, 1, N-1, y\right)\right. \\
\left.-\bar{\Xi}\left(-\frac{\beta_{m+1}}{\breve{\gamma}}, \dot{\gamma}, \bar{\gamma}, 1, N-1, y\right)\right]+\sum_{n=1}^{N-1} \sum_{k=0}^{n-1} \frac{B_{n}}{k ! \tilde{\gamma}^{k}} \\
.\left[\beta_{m}^{k} \bar{\Xi}\left(-\frac{\beta_{m}}{\widetilde{\gamma}}, \ddot{\gamma}, \bar{\gamma}, n-k, N-n+k, y\right)\right. \\
\left.-\beta_{m+1}^{k} \bar{\Xi}\left(-\frac{\beta_{m+1}}{\tilde{\gamma}}, \ddot{\gamma}, \bar{\gamma}, n-k, N-n+k, y\right)\right],
\end{array}
$$

where the function $\bar{\Xi}(a, b, c, p, q, y)$ is defined as the integral of the function $\Xi(a, b, c, p, q, y)$ :

$$
\bar{\Xi}(a, b, c, p, q, y)=\int_{-\infty}^{y} \Xi(a, b, c, p, q, y) d y,
$$

where $\Xi(a, b, c, p, q)$ is the back-transform of the generic term $e^{a((1-i \omega b) /(1-i \omega c))} /(1-i \omega b)^{p}(1-i \omega c)^{q}$ :

$$
\begin{aligned}
& \Xi(a, b, c, p, q, y) \\
& \quad=\frac{1}{2 \pi} \int_{-\infty}^{\infty} \frac{e^{a((1-i \omega b) /(1-i \omega c))}}{(1-i \omega b)^{p}(1-i \omega c)^{q}} e^{-i \omega y} d(i \omega) .
\end{aligned}
$$

For convenience, it is possible to express the function $\bar{\Xi}(a, b, c, p, q, y)$ as the contribution of two functions:

$$
\begin{aligned}
\bar{\Xi}(a, b, c, p, q, y)=\bar{U}(a, b, c, p, q, y) \\
+\bar{W}(a, b, c, p, q, y),
\end{aligned}
$$

where the functions $\bar{U}(\cdot)$ and $\bar{W}(\cdot)$ can be written (see Appendix D for details) as a series expansion:

$$
\begin{aligned}
\bar{U} & (a, b, c, p, q, y) \\
& =\sum_{r=0}^{p} \frac{a^{r}}{r !}\left(\sum_{j=1}^{p-r j-1} \sum_{s=0}^{-y / b} \frac{I_{j}^{(r)} y^{s}}{b^{s} s !}+\sum_{t=1}^{q+r t-1} \sum_{s=0}^{-y / c} \frac{K_{t}^{(r)} y^{s}}{c^{s} s !}\right),
\end{aligned}
$$

where the coefficients $I_{j}^{(r)}$ and $K_{t}^{(r)}$ are defined in expressions (D.10) and (D.11), respectively, in Appendix, and

$$
\begin{aligned}
\bar{W}(a, b, c, p, q, y) \\
=\sum_{r=p+1}^{\infty} \frac{a^{r}}{r !} \sum_{l=0}^{r-p}\left(\begin{array}{c}
r-p \\
l
\end{array}\right) b^{l} \\
\quad \times \sum_{k=0}^{l}\left(\begin{array}{l}
l \\
k
\end{array}\right) \sum_{s=0}^{q+r-1-k} \frac{(-1)^{k+l} y^{s}}{c^{l+s} s !} e^{-y / c} .
\end{aligned}
$$

It is now possible to write the probability term $\operatorname{Pr}\left\{\Gamma>\beta_{m} \mid\right.$ $\left.\beta_{m} \leq \widehat{\Gamma}<\beta_{m+1}\right\}$ as follows:

$$
\operatorname{Pr}\left\{\Gamma>\beta_{m} \mid \beta_{m} \leq \widehat{\Gamma}<\beta_{m+1}\right\}=\bar{F}_{\Gamma \mid \beta_{m} \leq \widehat{\Gamma}<\beta_{m+1}}\left(\beta_{m}\right) .
$$

The expressions in (17) and (29) can be substituted back in (8) to obtain the desired expression for the link-layer throughput.

\section{Results}

5.1. CDF Analysis. The results in Figure 3 display the cumulative distribution function (CDF) of the instantaneous SNR $\Gamma$ of the MRC receiver using the expression in (23) conditional on the estimated SNR $\widehat{\Gamma}$ exceeding a hypothetical reception threshold $\beta=2(\widehat{\Gamma}>2)$. The purpose of this setting is to study the statistics of the instantaneous SNR $\Gamma$ in terms of the MCS selection process based on imperfect (outdated) CSIT. The results have been obtained by using different values of the spatial and temporal correlation coefficients: $\rho=0.2,0.95$ and $\rho_{c}=0.2,0.95$.

For convenience in the discussion and visualization of results, a fixed value for average transmitting power settings $P / \sigma_{v}^{2}=1$ was used. Two values for the number of antennas were considered: $N=2$ and $N=4$. The first case $(N=2, \rho=$ $\left.0.2, \rho_{c}=0.2\right)$ in Figure 3 also presents the results of simulation work, showing a perfect match with the theoretical model, thus validating the proposed expressions. The results show an interesting behaviour. At low values of the $x$-axis, the results indicate, as expected, that the CDF for low values of the spatial correlation coefficient $(\rho \rightarrow 0)$ has better performance than the CDF for values of $\rho$ closer to one. However, this behaviour changes for larger values of the $x$-axis, where we can observe that higher values of spatial correlation $(\rho \rightarrow 1)$ provide better performance. Higher values of the $x$-axis correspond to a system where the target SNR of a given MCS is relatively high in comparison with the operational average SNR of 

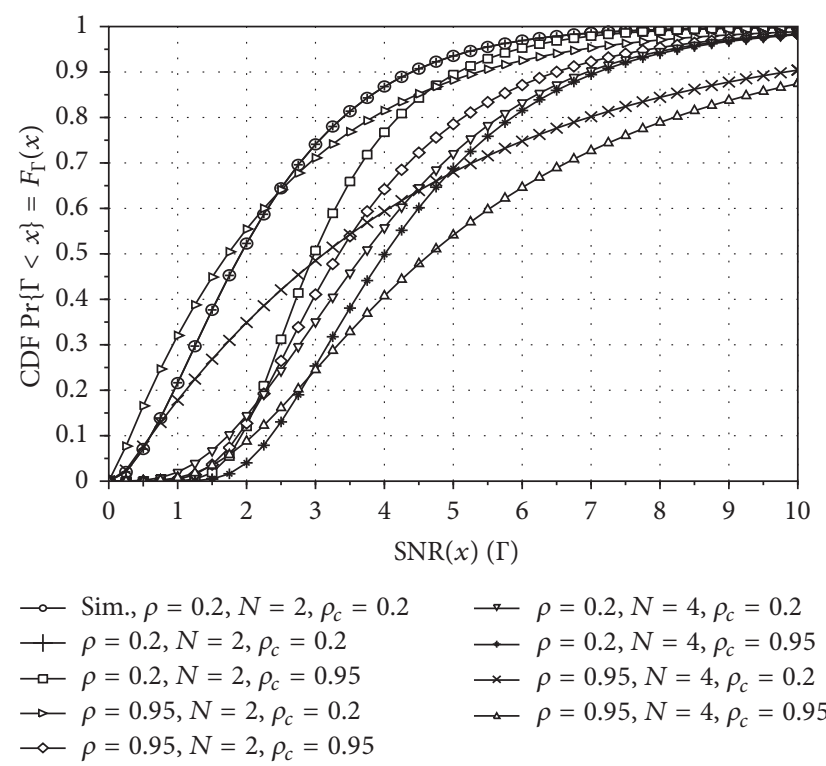

$\rightarrow \rho=0.2, N=4, \rho_{c}=0.2$

$\rightarrow \rho=0.2, N=4, \rho_{c}=0.95$

$\star \quad \rho=0.95, N=4, \rho_{c}=0.2$

$\rightarrow \rho=0.95, N=4, \rho_{c}=0.95$

FIGURE 3: CDF of the instantaneous SNR $\Gamma$ conditional on $\widehat{\Gamma}>2$ for different values of spatial and temporal correlation coefficients and number of antennas of the MRC receiver.

the system. This means that in the low SNR regime channel correlation can actually outperform the case of uncorrelated branches. At high SNR values, however, the performance of uncorrelated channels becomes clearly dominant. This behaviour can be explained by the fact that, at low SNR, the MRC receiver can be regarded as a simple averaging operation of noisy signals. Therefore, channel correlation becomes more useful for achieving a more accurate average of the received signal above the background noise. By contrast, at high SNR, the averaging operation over noise becomes less relevant, and the MRC operation obtains full benefit from uncorrelated signals.

The effects of imperfect CSIT on the CDF can be clearly observed as the curves in Figure 3 spread to the left of the hypothetical SNR decision threshold $\beta=2$. The larger the spreading of the CDF to the left of this threshold, the larger the error due to imperfect CSIT. We recall that the system is supposed to allow MCS transmission when the estimated SNR exceeds the target threshold $\beta$. Imperfect CSIT causes that the instantaneous SNR is not precisely always above the selection threshold, thus causing the spreading of the CDF as observed in Figure 3. The value of the CDF at $\widehat{\Gamma}=2$ is thus a measure of the error in the MCS selection process: it denotes the probability that the instantaneous SNR is below the threshold $\beta=2(\Gamma<\beta)$ when the estimated SNR was actually above this threshold $(\widehat{\Gamma} \geq \beta)$. We can observe how the CDF curves with higher correlation coefficient $\rho_{c}$ tend to get closer to the limit of the estimated SNR threshold $\beta=2$. Note that, with perfect CSIT $\left(\rho_{c}=1\right)$, the CDF of the instantaneous SNR only exists for values higher than $\beta=2(\Gamma>2)$. The figures also show that when the number of antennas is higher, the effects of imperfect CSIT tend to be reduced for low values of channel correlation. Higher number of antennas means higher diversity gains, which is translated into a higher probability of correct packet reception, even in the presence of an inaccurate CSIT for MCS selection. However, the opposite behaviour is observed for larger values of spatial correlation, which tend to increase the probability of decision error due to imperfect CSIT, particularly when the number of antennas increases. Channel correlation tends to reduce the diversity of the receiver, and therefore it also reduces the probability of the instantaneous SNR to surpass the MCS detection threshold in the presence of imperfect CSIT. In addition, as the number of antennas increases, the lack of diversity due to channel correlation accumulates, leading to higher probabilities of error in the MCS assignment due to imperfect CSIT. As a consequence of this, it can be also observed that the effects of the dominance of correlated channels for low SNR values tend to be reduced with imperfect CSIT, particularly when the number of antennas increases.

From Figure 3 it is also possible to observe that the error of MCS selection could be potentially reduced by setting a decision threshold slightly higher than the target threshold of the selected MCS. However, this also reduces the probability of MCS selection, which could be actually worse in terms of capacity than the gain provided by the reduction of error of MCS selection. On the other hand, the receiver can be potentially affected by unknown cochannel interference, a scenario where setting a slightly higher decision threshold might lead to further gains to overcome the reduction of the probability of MCS selection. The investigation of this effect remains open for future research works considering cochannel interference.

It is also possible to observe in the formulas for CCDF in (23) that as the correlation coefficient of the estimated CSI $\rho_{c}$ becomes zero $\left(\rho_{c} \rightarrow 0\right)$, the adaptation scheme becomes less relevant and degrades into a random MCS assignment scheme. This is because the correlation model in (5) indicates that when the correlation coefficient is set to zero $\left(\rho_{c}=0\right)$, the adaptation scheme has no knowledge of the instantaneous channel outcome, but it still has knowledge of the second-order statistics of the channel (average channel gain). Therefore, random MCS assignment based on the knowledge of the average channel gain is the optimum allocation scheme. If the scheduler has no knowledge of this average channel gain, then the MCS assignment process becomes meaningless. This agrees with the main idea behind MCS adaptation, whose main advantage only depends on the ability of the system to accurately track instantaneous channel variations and adapt accordingly.

5.2. WiMAX Modulation. This subsection employs the analytic formulae derived in previous sections to study a full wireless transmission system (WiMAX) with several MCSs taken from a LUT that has been previously obtained via PHYlayer simulation. The MCSs used correspond to the WiMAX standard in [38], summarized in Table 1, using a block length of 7200 symbols $(Q=7200)$. The spectral efficiency of each MCS is obtained by using the number of bits per constellation times the code rate displayed in Table 1 and other parameters such as frame length and repetition code rate as presented in 
TABLE 1: WiMAX modulation and coding schemes [38].

(a)

\begin{tabular}{lccccc}
\hline & QPSK $1 / 3$ & & QPSK 1/2 & & QPSK 2/3 \\
SINR & BLER & SINR & BLER & SINR & BLER \\
\hline-1.14 & $4.10 e-3$ & 1.32 & $4.13 e-3$ & 3.47 & $6.50 e-3$ \\
\hline
\end{tabular}

(b)

\begin{tabular}{lcccccc}
\hline & QPSK 3/4 & \multicolumn{2}{c}{ QPSK 4/5 } & & 16 QAM 1/3 \\
SINR & BLER & SINR & BLER & SINR & BLER \\
\hline 4.78 & $3.30 e-3$ & 5.46 & $4.97 e-3$ & 3.66 & $7.15 e-3$ \\
\hline
\end{tabular}

(c)

\begin{tabular}{lccccc}
\hline & 16 QAM $1 / 2$ & & 16 QAM 2/3 & & 16 QAM 3/4 \\
SINR & BLER & SINR & BLER & SINR & 1.98 \\
\hline 6.52 & $5.70 e-3$ & 9.37 & $3.80 e-3$ & BLER \\
\hline
\end{tabular}

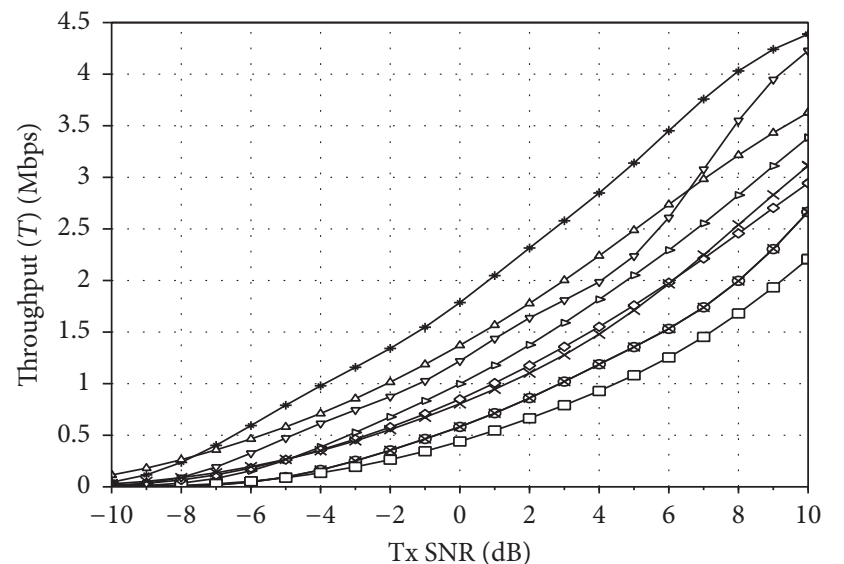

$$
\begin{array}{ll}
\multimap \text { Sim., } \rho=0.2, N=2, \rho_{c}=0.2 & \rightarrow-\rho=0.2, N=4, \rho_{c}=0.2 \\
\rightarrow \rho=0.2, N=2, \rho_{c}=0.2 & \rightarrow \rho=0.2, N=4, \rho_{c}=0.95 \\
\rightarrow-\rho=0.2, N=2, \rho_{c}=0.95 & \rightarrow-\rho=0.95, N=4, \rho_{c}=0.2 \\
\multimap-\rho=0.95, N=2, \rho_{c}=0.2 & \rightarrow-\rho=0.95, N=4, \rho_{c}=0.95
\end{array}
$$$$
\neg \rho=0.95, N=2, \rho_{c}=0.95
$$

FIGURE 4: Throughput versus transmitting SNR $\left(P \gamma / \sigma_{v}^{2}\right)$ in $\mathrm{dB}$ for different values of spatial and temporal correlation coefficients and number of antennas of the MRC receiver.

the original standard in [38]. Note that the SNR thresholds displayed in Table 1 are in dBs. Figure 4 shows the link-layer throughput calculated by using the expression in (8) versus the transmitting power to noise ratio settings $P \gamma / \sigma_{v}^{2}$ using different values of the correlation coefficients $\left(\rho\right.$ and $\left.\rho_{c}\right)$ and different numbers of antennas of the MRC receiver.

The first case $\left(N=2, \rho=0.2\right.$, and $\left.\rho_{c}=0.2\right)$ in Figure 4 also presents the results of simulation work, showing a perfect match with the theoretical model. The results show that at high SNR the total throughput improves, particularly when spatial correlation coefficient is reduced and also by increasing the numbers of the antennas of the MRC receiver. However, performance is generally degraded in case of imperfect CSIT (lower values of $\rho_{c}$ ). It can be observed that, at low SNR, the results behave differently: high spatial correlation values lead to higher throughput. This confirms the results presented in the previous subsection. The degrading effects of imperfect CSIT can be observed to be enhanced in the high SNR regime as the values of spatial correlation and the number of antennas increase. Finally, the region of SNR values for which spatial correlated performance is shown to outperform the uncorrelated case is observed to be considerably reduced for larger values of the number of antennas, while imperfect CSIT has a negative but almost imperceptible effect on this low SNR behaviour as displayed in Figure 4. This slight difference with respect to the results in the previous subsection is mainly due to the particular MCSs being used and the WiMAX system settings.

\section{Conclusions}

A performance model of the packet reception process of AMC-MRC receiver with spatially correlated channels and imperfect CSIT was proposed. This is the first work where spatial correlation and limited feedback are considered in the same analysis of adaptive MRC receivers. The reception model is based on the derivation of analytic expressions of correct packet reception probabilities in Rayleigh fading channels as an extension of outage probability analysis and SNR switching threshold selection. The model is useful for AMC systems, where the correct reception probability of each MCS is weighted by the operational BLER and spectral efficiency considering operation at the SNR threshold of each MCS. The proposed model provides a flexible abstraction of the adaptive PHY-layer that can be used in the optimization and design of upper layers. The results point towards an interesting conclusion: spatial channel correlation is not always bad for capacity. In the low SNR regime it can provide higher gains than the case of spatially uncorrelated channel. In the high SNR regime it was confirmed that uncorrelated channels provide more capacity gains than the correlated case. Imperfect CSIT was shown to reduce performance due to decision errors in MCS assignment. The degrading effects 
of imperfect CSIT were found to be enhanced by channel spatial correlation and by higher numbers of antennas. Correlation was found to reduce diversity gains of the receiver that in turn reduce the probability of correct packet reception in the presence of imperfect CSIT. This effect was found to be enhanced by increasing the number of antennas as correlation degrading effects tend to be amplified (lack of diversity is accumulated over the antennas). Finally, the dominance of correlated channels was found to be reduced with imperfect CSIT and with an increase of the number of antennas.

\section{Appendix}

\section{A. Derivation of the Unconditional CF of $\widehat{\Gamma}_{j}$ in (13)}

Consider the following modification of (12):

$$
\begin{aligned}
& \Psi_{\widehat{\Gamma}}(i \omega) \\
& \quad=\int_{0}^{\infty}(1-i \omega \widetilde{\gamma})^{-N} \gamma^{-1} e^{-x((1-i \omega(\alpha \gamma+\widetilde{\gamma})) / \gamma(1-i \omega \widetilde{\gamma}))} d x .
\end{aligned}
$$

By using the following changes of variable $u=x((1-i \omega(\alpha \gamma+$ $\tilde{\gamma})) / \gamma(1-i \omega \tilde{\gamma})), \breve{\gamma}=\alpha \gamma+\widetilde{\gamma}$, and $d u=d x((1-i \omega(\alpha \gamma+\widetilde{\gamma})) / \gamma(1-$ $i \omega \widetilde{\gamma}))$ the previous integral becomes

$$
\Psi_{\widehat{\Gamma}}(i \omega)=\int_{0}^{\infty}(1-i \omega \widetilde{\gamma})^{1-N}(1-i \omega \breve{\gamma})^{-1} e^{-u} d u,
$$

which after the integration $\left(\int_{u=0}^{\infty} e^{-u} d u=1\right)$ yields the expression in (13).

\section{B. Derivation of Coefficients $A$ and $B_{n}$ in (14)}

Consider the following normalized version of the expression for the unconditional CF of the instantaneous SNR of the MRC receiver in (13):

$$
\Psi_{\widehat{\Gamma}}(i \omega)
$$

$$
=(-\breve{\gamma})^{-1}(-\widetilde{\gamma})^{1-N}\left(i \omega-\frac{1}{\breve{\gamma}}\right)^{-1}\left(i \omega-\frac{1}{\widetilde{\gamma}}\right)^{1-N} .
$$

This expression can be rewritten as a standard PFE problem:

$$
\begin{aligned}
& \Psi_{\widehat{\Gamma}}(i \omega) \\
& \quad=(-\breve{\gamma})^{-1}(-\widetilde{\gamma})^{1-N}\left(\frac{\check{A}}{i \omega-1 / \breve{\gamma}}+\sum_{n=1}^{N-1} \frac{\check{B}_{n}}{(i \omega-1 / \tilde{\gamma})^{n}}\right),
\end{aligned}
$$

where $\check{A}$ and $\check{B}_{n}$ are the coefficients of a standard PFE problem with repeated roots:

$$
\begin{aligned}
& \check{A}=\left(\frac{1}{\breve{\gamma}}-\frac{1}{\widetilde{\gamma}}\right)^{1-N}, \\
& \check{B}_{n}=\left(\frac{1}{\tilde{\gamma}}-\frac{1}{\breve{\gamma}}\right)^{n-N} .
\end{aligned}
$$

It is now possible to rewrite the expression in (B.2) back in a nonstandard notation as follows:

$$
\Psi_{\widehat{\Gamma}}(i \omega)=\frac{\check{A}(-\widetilde{\gamma})^{1-N}}{1-i \omega \check{\gamma}}+\sum_{n=1}^{N-1} \frac{\check{B}_{n}(-\breve{\gamma})^{-1}(-\tilde{\gamma})^{n+1-N}}{(1-i \omega \tilde{\gamma})^{n}}
$$

which can be rewritten as the expression in (14):

$$
\Psi_{\widehat{\Gamma}}(i \omega)=A(1-i \omega \breve{\gamma})^{-1}+\sum_{n=1}^{N-1} B_{n}(1-i \omega \widetilde{\gamma})^{-n} .
$$

By comparing these two last expressions it follows that

$$
\begin{aligned}
A & =\check{A}(-\widetilde{\gamma})^{1-N}=\left(1-\frac{\widetilde{\gamma}}{\breve{\gamma}}\right)^{1-N}, \\
B_{n} & =\check{B}_{n}(-\breve{\gamma})^{-1}(-\widetilde{\gamma})^{n+1-N}=\left(-\frac{\widetilde{\gamma}}{\breve{\gamma}}\right)\left(1-\frac{\widetilde{\gamma}}{\breve{\gamma}}\right)^{n-N},
\end{aligned}
$$

which completes the derivation of the coefficients in (14).

\section{Derivation of the CF of the Instantaneous SNR $\Gamma$ in (22) Conditional on $\beta_{m} \leq \widehat{\Gamma}<\beta_{m+1}$}

The CF of the instantaneous SNR conditional on the estimated SNR being in the range $\beta_{m} \leq \widehat{\Gamma}<\beta_{m+1}$ can be calculated as in (21):

$$
\begin{aligned}
& \Psi_{\Gamma \mid \beta_{m} \leq \widehat{\Gamma}<\beta_{m+1}} \\
& \quad=\int_{\beta_{m}}^{\beta_{m+1}}(1-i \omega \bar{\gamma})^{-N} e^{i \omega \rho_{c}^{2} \widehat{\Gamma} /(1-i \omega \bar{\gamma})} f_{\widehat{\Gamma}}(\widehat{\Gamma}) d \widehat{\Gamma} .
\end{aligned}
$$

By substituting the PDF of the estimated SNR taken from (15) in the previous expression, we obtain

$$
\begin{gathered}
\Psi_{\Gamma \mid \beta_{m} \leq \widehat{\Gamma}<\beta_{m+1}}(i \omega)=\int_{y=\beta_{m}}^{y=\beta_{m+1}}(1-i \omega \bar{\gamma})^{-N} e^{i \omega \rho_{c}^{2} y /(1-i \omega \bar{\gamma})} \\
\cdot \frac{A}{\breve{\gamma}} e^{-y / \breve{\gamma}} d y+\int_{\beta_{m}}^{\beta_{m+1}}(1-i \omega \bar{\gamma})^{-N} \\
\cdot e^{i \omega \rho_{c}^{2} y /(1-i \omega \bar{\gamma})} e^{-y / \tilde{\gamma}} \sum_{n=1}^{N-1} \frac{B_{n} y^{n-1}}{\tilde{\gamma}^{n}(n-1) !} d y .
\end{gathered}
$$

Now consider a modification of the first integral in (C.2) and by using the change of variable $\dot{\gamma}=\rho_{c}^{2} \breve{\gamma}+\bar{\gamma}$ we obtain

$$
\int_{\beta_{m}}^{\beta_{m+1}}(1-i \omega \bar{\gamma})^{-N} e^{-y((1-i \omega \dot{\gamma}) / \breve{\gamma}(1-i \omega \bar{\gamma}))} \frac{A}{\breve{\gamma}} d y,
$$

which after integration becomes

$$
\frac{A\left(e^{-\beta_{m}(1-i \omega \dot{\gamma}) / \breve{\gamma}(1-i \omega \bar{\gamma})}-e^{-\beta_{m+1}(1-i \omega \dot{\gamma}) / \breve{\gamma}(1-i \omega \bar{\gamma})}\right)}{(1-i \omega \dot{\gamma})(1-i \omega \bar{\gamma})^{N-1}} .
$$


Consider now a modification of the second term of the integral in (C.2), and by using the change of variable $\ddot{\gamma}=$ $\rho_{c}^{2} \tilde{\gamma}+\bar{\gamma}$, we obtain

$$
\begin{aligned}
& \int_{\beta_{m}}^{\beta_{m+1}}(1-i \omega \bar{\gamma})^{-N} \\
& \quad \cdot e^{-y((1-i \omega \ddot{\gamma}) / \tilde{\gamma}(1-i \omega \bar{\gamma}))} \sum_{n=1}^{N-1} \frac{B_{n} y^{n-1}}{\widetilde{\gamma}^{n}(n-1) !} d y,
\end{aligned}
$$

which after the integration becomes:

$$
\sum_{n=1}^{N-1} \sum_{u=0}^{n-1} \frac{B_{n}\left(\beta_{m}^{u} e^{-\beta_{m}(1-i \omega \ddot{\gamma}) / \widetilde{\gamma}(1-i \omega \bar{\gamma})}-\beta_{m+1}^{u} e^{-\beta_{m+1}(1-i \omega \ddot{\gamma}) / \widetilde{\gamma}(1-i \omega \bar{\gamma})}\right)}{u ! \tilde{\gamma}^{u}(1-i \omega \ddot{\gamma})^{n-u}(1-i \omega \bar{\gamma})^{N-n+u}} .
$$

The term in (C.4) together with the term in the previous expression (C.6) constitutes the solution of the integral in (C.1) and (C.2), which is the desired expression in (22).

\section{Derivation of $\bar{\Xi}(a, b, c, p, q)$ in (26)}

Consider the generic term used in the definition of $\Xi(a, b, c, p, q)$ in (25). Using the Taylor series expansion of this term we can obtain the following:

$$
\begin{aligned}
& \frac{e^{a((1-i \omega b) /(1-i \omega c))}}{(1-i \omega b)^{p}(1-i \omega c)^{q}} \\
& \quad=\sum_{r=0}^{p} \frac{a^{r}}{r !} \frac{(1-i \omega b)^{r-p}}{(1-i \omega c)^{q+r}}+\sum_{r=p+1}^{\infty} \frac{a^{r}}{r !} \frac{(1-i \omega b)^{r-p}}{(1-i \omega c)^{q+r}} .
\end{aligned}
$$

The following subsections focus on the derivation of the two terms in the previous expression and their respective backtransform functions, which are defined as follows:

$$
\begin{aligned}
U(\cdot) & =\frac{1}{2 \pi} \int_{\omega}\left(\sum_{r=0}^{p} \frac{a^{r}}{r !} \frac{(1-i \omega b)^{r-p}}{(1-i \omega c)^{q+r}}\right) e^{-i \omega y} d(i \omega), \\
W(\cdot) & =\frac{1}{2 \pi} \int_{\omega}\left(\sum_{r=p+1}^{\infty} \frac{a^{r}}{r !} \frac{(1-i \omega b)^{r-p}}{(1-i \omega c)^{q+r}}\right) e^{-i \omega y} d(i \omega) .
\end{aligned}
$$

Note that under these definitions we can provide an expression for the function $\Xi(a, b, c, p, q)$ as given in (26), which is the summation of the functions defined by (D.2) and (D.3):

$$
\begin{aligned}
\Xi(a, b, c, p, q, y)= & U(a, b, c, p, q, y) \\
& +W(a, b, c, p, q, y) .
\end{aligned}
$$

Similarly, the CCDF of the function $\Xi(a, b, c, p, q)$, denoted by $\bar{\Xi}(a, b, c, p, q)$, will be expressed as follows:

$$
\begin{aligned}
\bar{\Xi}(a, b, c, p, q, y)= & \bar{U}(a, b, c, p, q, y) \\
& +\bar{W}(a, b, c, p, q, y)
\end{aligned}
$$

where $\bar{U}(\cdot)=\int_{0}^{y} U(\cdot) d y$ and $\bar{W}(\cdot)=\int_{0}^{y} W(\cdot) d y$ are the CCDF components of the terms $U(\cdot)$ and $W(\cdot)$, respectively.

Case $1(r \leq p)$.

Derivation of (D.2). In the case when $r \leq p$, which corresponds to the first term of the expression in (D.1), it is possible to rewrite each of the terms of the summation using PFE as follows:

$$
\begin{aligned}
& \frac{1}{(1-i \omega b)^{p-r}(1-i \omega c)^{q+r}} \\
& \quad=\sum_{j=1}^{p-r} \frac{I_{j}^{(r)}}{(1-i \omega b)^{j}}+\sum_{t=1}^{q+r} \frac{K_{t}^{(r)}}{(1-i \omega c)^{t}} .
\end{aligned}
$$

For convenience, let us rewrite the previous equation as a standard PFE problem:

$$
\begin{aligned}
\frac{1}{(1-i \omega b)^{p-r}(1-i \omega c)^{q+r}} \\
=\frac{(-b)^{r-p}(-c)^{-q-r}}{(i \omega-1 / b)^{p-r}(i \omega-1 / c)^{q+r}}=(-b)^{r-p}(-c)^{-q-r} \\
\quad\left(\sum_{j=1}^{p-r} \frac{\check{I}_{j}^{(r)}}{(i \omega-1 / b)^{j}}+\sum_{t=1}^{q+r} \frac{\check{K}_{t}^{(r)}}{(i \omega-1 / c)^{t}}\right),
\end{aligned}
$$

where $\check{I}_{j}^{(r)}$ and $\check{K}_{t}^{(r)}$ are the coefficients of a standard PFE problem with repeated roots:

$$
\begin{aligned}
& \check{I}_{j}^{(r)}=(-1)^{p-r-j}\left(\begin{array}{c}
q+p-j-1 \\
p-r-j
\end{array}\right)\left(\frac{1}{b}-\frac{1}{c}\right)^{-q-p+j}, \\
& \check{K}_{t}^{(r)}=(-1)^{q+r-t}\left(\begin{array}{c}
p+q-t-1 \\
q+r-t
\end{array}\right)\left(\frac{1}{c}-\frac{1}{b}\right)^{-p-q+t} .
\end{aligned}
$$

It is now possible to rewrite (D.7) back in not standard form:

$$
\begin{aligned}
& \sum_{j=1}^{p-r} \frac{\check{I}_{j}^{(r)}(-b)^{r-p+j}(-c)^{-q-r}}{(1-i \omega b)^{j}} \\
& +\sum_{t=1}^{q+r} \frac{\check{K}_{t}^{(r)}(-b)^{r-p}(-c)^{-q-r+t}}{(1-i \omega c)^{t}}
\end{aligned}
$$

which is similar to the expression in (D.6). By comparing this last expression with (D.6) we obtain the following expressions for the coefficients $I_{j}^{(r)}$ and $K_{t}^{(r)}$ :

$$
\begin{aligned}
I_{j}^{(r)} & =\check{I}_{j}^{(r)}(-b)^{r-p+j}(-c)^{-q-r}, \\
K_{t}^{(r)} & =\check{K}_{t}^{(r)}(-b)^{r-p}(-c)^{-q-r+t} .
\end{aligned}
$$


It is now possible to find the first summation term in (D.1):

$$
\begin{aligned}
\sum_{r=0}^{p} & \frac{a^{r}}{r !} \frac{(1-i \omega b)^{r-p}}{(1-i \omega c)^{q+r}} \\
& =\sum_{r=0}^{p} \frac{a^{r}}{r !}\left(\sum_{j=1}^{p-r} \frac{I_{j}^{(r)}}{(1-i \omega b)^{j}}+\sum_{t=1}^{q+r} \frac{K_{t}^{(r)}}{(1-i \omega c)^{t}}\right),
\end{aligned}
$$

whose back-transform yields to the following explicit expression for the function in (D.2):

$$
\begin{aligned}
& U(\cdot) \\
& =\sum_{r=0}^{p} \frac{a^{r}}{r !}\left(\sum_{j=1}^{p-r} e^{-y / b} \frac{I_{j}^{(r)} y^{j-1}}{b^{j} j !}+\sum_{t=1}^{q+r} e^{-y / c} \frac{K_{t}^{(r)} y^{t-1}}{c^{t} t !}\right) .
\end{aligned}
$$

The CCDF of this expression $\left(\bar{U}(\cdot)=\int_{y}^{\infty} U(\cdot) d y\right)$ can be written as

$$
\begin{aligned}
& U(\cdot) \\
& =\sum_{r=0}^{p} \frac{a^{r}}{r !}\left(\sum_{j=1}^{p-r} \sum_{s=0}^{j-1} e^{-y / b} \frac{I_{j}^{(r)} y^{s}}{b^{s} s !}+\sum_{t=1}^{q+r} \sum_{s=0}^{t-1} e^{-y / c} \frac{K_{t}^{(r)} y^{s}}{c^{s} s !}\right) .
\end{aligned}
$$

Case $2(r>p)$.

Derivation of (D.3). Consider the expansion of the second term in (D.1):

$$
\begin{aligned}
& \sum_{r=p+1}^{\infty} \frac{a^{r}}{r !} \frac{(1-i \omega b)^{r-p}}{(1-i \omega c)^{q+r}} \\
& \quad=\sum_{r=p+1}^{\infty} \frac{a^{r}}{r !} \sum_{l=0}^{r-p}\left(\begin{array}{c}
r-p \\
l
\end{array}\right) \frac{(-i \omega b)^{l}}{(1-i \omega c)^{q+r}} .
\end{aligned}
$$

By using the properties of the Fourier transform analysis, the back-transform of this last expression is given by

$$
\begin{aligned}
& W(\cdot)=\sum_{r=p+1}^{\infty} \frac{a^{r}}{r !} \sum_{l=0}^{r-p}\left(\begin{array}{c}
r-p \\
l
\end{array}\right) \\
& \cdot(-b)^{l} \frac{d^{l}}{d y^{l}}\left(\frac{y^{q+r-1}}{c^{q+r}(q+r) !} e^{-y / c}\right)=\sum_{r=p+1}^{\infty} \frac{a^{r}}{r !} \\
& \cdot \sum_{l=0}^{r-p}\left(\begin{array}{c}
r-p \\
l
\end{array}\right)(-b)^{l} \times \sum_{k=0}^{l}\left(\begin{array}{l}
l \\
k
\end{array}\right) \\
& \cdot \frac{(-1)^{k} y^{q+r-1-k}}{c^{q+r+l-k}(q+r-1-k) !} e^{-y / c} .
\end{aligned}
$$

The CCDF of this expression $\left(\bar{W}(\cdot)=\int_{y}^{\infty} W(\cdot) d y\right)$ can be written as

$$
\begin{aligned}
\bar{W}(\cdot)= & \sum_{r=p+1}^{\infty} \frac{a^{r}}{r !} \sum_{l=0}^{r-p}\left(\begin{array}{c}
r-p \\
l
\end{array}\right) b^{l} \\
& \times \sum_{k=0}^{l}\left(\begin{array}{l}
l \\
k
\end{array}\right) \sum_{s=0}^{q+r-1-k} \frac{(-1)^{k+l} y^{s}}{c^{l+s} s !} e^{-y / c} .
\end{aligned}
$$

\section{Competing Interests}

The authors declare that they have no competing interests.

\section{Acknowledgments}

The study was funded by FCT/MEC (Fundação para a Ciência e a Tecnologia), ERDF (European Regional Development Fund) under PT2020, CISTER Research Unit (CEC/04234), and ARTEMIS/0004/2013-JU Grant no. 621353 (DEWI, http://www.dewi-project.eu).

\section{References}

[1] L. Tong, V. Naware, and P. Venkitasubramaniam, "Signal processing in random access," IEEE Signal Processing Magazine, vol. 21, no. 5, pp. 29-39, 2004.

[2] G. Dimić, N. D. Sidiropoulos, and R. Zhang, "Medium access control-physical cross-layer design," IEEE Signal Processing Magazine, vol. 21, no. 5, pp. 40-50, 2004.

[3] V. Srivastava and M. Motani, "Cross-layer design: a survey and the road ahead," IEEE Communications Magazine, vol. 43, no. 12, pp. 112-119, 2005.

[4] M. H. Ngo and V. Krishnamurthy, "Game theoretic crosslayer transmission policies in multipacket reception wireless networks," IEEE Transactions on Signal Processing, vol. 55, no. 5, pp. 1911-1926, 2007.

[5] Y. Zhou, J. Liu, C. Zhai, and L. Zheng, "Two-transmitter tworeceiver cooperative MAC protocol: cross-layer design and performance analysis," IEEE Transactions on Vehicular Technology, vol. 59, no. 8, pp. 4116-4127, 2010.

[6] W. Chen, L. Dai, K. B. Letaief, and Z. Cao, "A unified cross-layer framework for resource allocation in cooperative networks," IEEE Transactions on Wireless Communications, vol. 7, no. 8, pp. 3000-3012, 2008.

[7] F. Baccelli, B. Błaszczyszyn, and P. Mühlethaler, "Stochastic analysis of spatial and opportunistic Aloha," IEEE Journal on Selected Areas in Communications, vol. 27, no. 7, pp. 1105-1119, 2009.

[8] S. Ghez, S. Verdu, and S. C. Schwartz, "Stability properties of slotted Aloha with multipacket reception capability," IEEE Transactions on Automatic Control, vol. 33, no. 7, pp. 640-649, 1988.

[9] V. Naware, G. Mergen, and L. Tong, "Stability and delay of finite-user slotted ALOHA with multipacket reception," IEEE Transactions on Information Theory, vol. 51, no. 7, pp. 26362656, 2005.

[10] Deliverable D5.1: System level evaluation metrics and interfacing, "FP7 CODIV: Enhanced Wireless Communication Systems Employing COoperative DIVersity", http://www.ict-codiv.eu/.

[11] Deliverable D5.4: Final report on link level and system level channel models, "FP7 WINNER: Wireless World Initiative New Radio," http://www.ist-winner.org.

[12] Deliverable D7.1: System level interfacing, metrics and simulation scenarios, "FP7 FUTON: Fibre-Optic Networks for Distributed Extendible Heterogeneous Radio Architectures and Service", http://www.ict-futon.eu/.

[13] K. Brueninghaus, D. Astélyt, T. Salzer et al., "Link performance models for system level simulations of broadband radio access 
systems," in Proceedings of the IEEE 16th International Symposium on Personal, Indoor and Mobile Radio Communications, (PIMRC '05), pp. 2306-2311, September 2005.

[14] J. Murkovic, G. Orfanos, and H. J. Reumermann, "MIMO link modeling for system-level simulations," in Proceedings of the 17th annual IEEE International Symposium on Personal, Indoor and Mobile Radio Communications (PMRC '06), vol. 4, pp. 1-6, 2006.

[15] M. Wrulich and M. Rupp, "Efficient link measurement model for system level simulations of Alamouti encoded MIMO HSDPA transmissions," in Proceedings of the International ITG Workshop on Smart Antennas (WSA '08), pp. 351-355, February 2008.

[16] A. Perez-Niera and M. Campalans, Cross-Layer Resource Allocation in Wireless Communications: Techniques and Models from PHY and MAC Layer Interaction, Academic Press, Oxford, UK, 2010.

[17] F. A. Dietrich and W. Utschick, "Maximum ratio combining of correlated Rayleigh fading channels with imperfect channel knowledge," IEEE Communications Letters, vol. 7, no. 9, pp. 419421, 2003.

[18] Y. Ma, R. Schober, and S. Pasupathy, "Effect of channel estimation errors on MRC diversity in Rician fading channels," IEEE Transactions on Vehicular Technology, vol. 54, no. 6, pp. 21372142, 2005.

[19] Y. Ma, "Impact of correlated diversity branches in Rician fading channels," in Proceedings of the IEEE International Conference on Communications (ICC '05), vol. 1, pp. 473-477, Seoul, South Korea, May 2005.

[20] H. T. Hui, "The performance of the maximum ratio combining method in correlated rician-fading channels for antennadiversity signal combining," IEEE Transactions on Antennas and Propagation, vol. 53, no. 3, pp. 958-964, 2005.

[21] P. Loskot and N. C. Beaulieu, "A unified approach to computing error probabilities of diversity combining schemes over correlated fading channels," IEEE Transactions on Communications, vol. 57, no. 7, pp. 2031-2041, 2009.

[22] N. C. Beaulieu and X. Zhang, "On selecting the number of receiver diversity antennas in ricean fading cochannel interference," in Proceedings of the IEEE Global Telecommunications Conference (GLOBECOM '06), pp. 1-6, San Francisco, Calif, USA, December 2006.

[23] N. C. Beaulieu and X. Zhang, "On the maximum number of receiver diversity antennas that can be usefully deployed in a cochannel interference dominated environment," IEEE Transactions on Signal Processing, vol. 55, no. 7, pp. 3349-3359, 2007.

[24] N. C. Beaulieu and X. Zhang, "On the maximum useful number of receiver antennas for MRC diversity in cochannel interference and noise," in Proceedings of the IEEE International Conference on Communications (ICC '07), pp. 5103-5108, IEEE, June 2007.

[25] K. S. Ahn and R. W. Heath Jr., "Performance analysis of maximum ratio combining with imperfect channel estimation in the presence of cochannel interferences," IEEE Transactions on Wireless Communications, vol. 8, no. 3, pp. 1080-1085, 2009.

[26] R. Samano-Robles and A. Gameiro, "Joint spectrum selection and radio resource management for distributed antenna systems with cognitive radio and space division multiplexing," in Proceedings of the 17th International ITG Workshop on Smart Antennas (WSA '13), Stuttgart, Germany, March 2013.
[27] L. Chen, Y. Yang, X. Chen, and G. Wei, "Energy-efficient link adaptation on rayleigh fading channel for OSTBC MIMO system with imperfect CSIT," IEEE Transactions on Vehicular Technology, vol. 62, no. 4, pp. 1577-1585, 2013.

[28] R. Samano-Robles and E. Lavendelis, "Performance model for MRC receivers with adaptive modulation and coding in rayleigh fading correlated channels with imperfect CSIT," in Proceedings of the Advances in Wireless and Optical Communications (RTUWO '15), pp. 26-29, Riga, Latvia, November 2015.

[29] R. Sámano-Robles and A. Gameiro, "A performance model for maximum ratio combining receivers with adaptive modulation and coding in Rice fading correlated channels," in Proceedings of the 19th IEEE Symposium on Computers and Communications (ISCC '14), Funchal, Portugal, June 2014.

[30] A. E. Ekpenyong and Y.-F. Huang, "Feedback constraints for adaptive transmission," IEEE Signal Processing Magazine, vol. 24, no. 3, pp. 69-78, 2007.

[31] S. Zhou and G. B. Giannakis, "Adaptive modulation for multiantenna transmissions with channel mean feedback," IEEE Transactions on Wireless Communications, vol. 3, no. 5, pp. 1626-1636, 2004.

[32] M. Torabi and J. F. Frigon, "Impact of outdated feedback on the performance of M-QAM adaptive modulation in user selection diversity systems with OSTBC over MIMO rayleigh fading channels," in Proceedings of the 2012 IEEE Vehicular Technology Conference (VTC Fall '12), pp. 1-5, Quebec City, Canada, 2012.

[33] P. Xia, S. Zhou, and G. B. Giannakis, "Multiantenna adaptive modulation with beamforming based on bandwidthconstrained feedback," IEEE Transactions on Communications, vol. 53, no. 3, pp. 526-536, 2005.

[34] Z. Bouida, A. Ghrayeb, and K. A. Qaraqe, "Adaptive spatial modulation for spectrum sharing systems with limited feedback," IEEE Transactions on Communications, vol. 63, no. 6, pp. 2001-2014, 2015.

[35] Z. Bouida, A. Ghrayeb, and K. A. Qaraqe, "Joint adaptive spatial modulation and power adaptation for spectrum sharing systems with limited feedback," in Proceedings of the IEEE Wireless Communications and Networking Conference (WCNC '15), pp. 789-794, IEEE, New Orleans, La, USA, March 2015.

[36] P. Yang, Y. Xiao, Y. Yu, L. Li, Q. Tang, and S. Li, "Simplified adaptive spatial modulation for limited-feedback MIMO systems," IEEE Transactions on Vehicular Technology, vol. 62, no. 6, pp. 2656-2666, 2013.

[37] J. Proakis, Digital Communications, McGraw-Hill, 4th edition, 2001.

[38] WiMAX Forum Standard, "WiMAX system level evaluation methodology, V.0.0.1, 2006. 


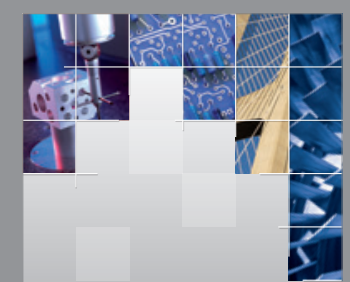

\section{Enfincering}
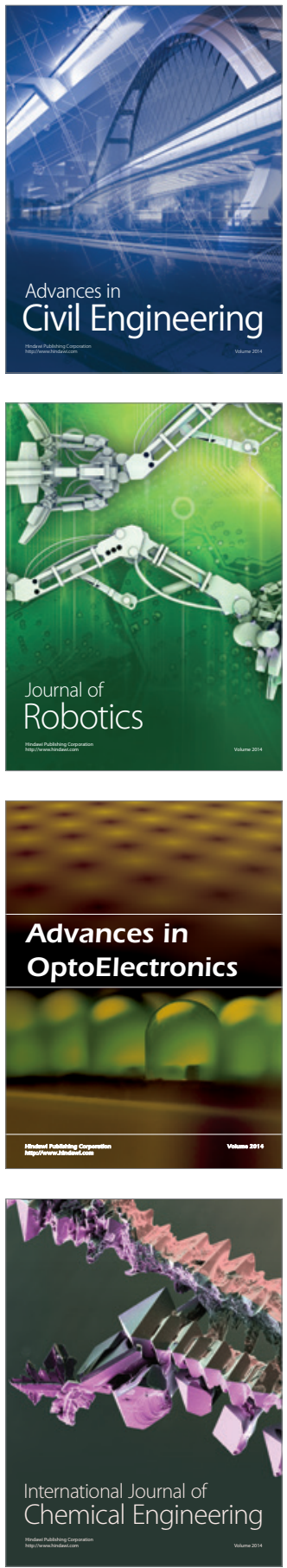

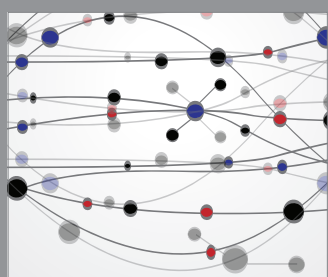

The Scientific World Journal

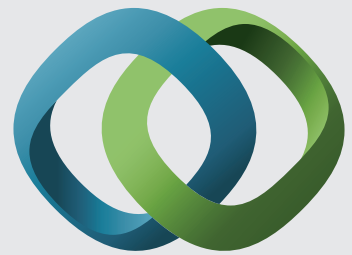

\section{Hindawi}

Submit your manuscripts at

https://www.hindawi.com
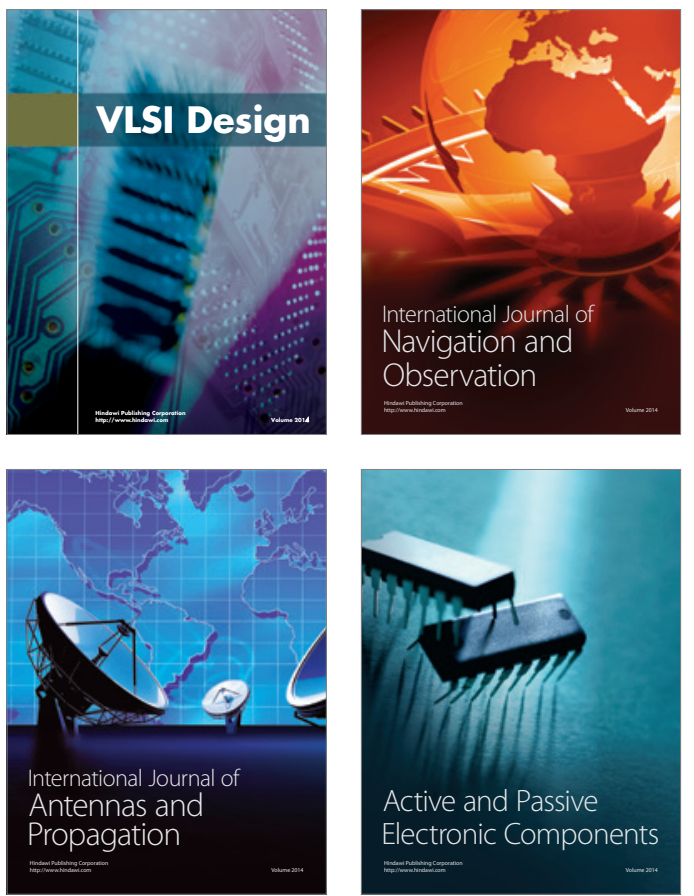
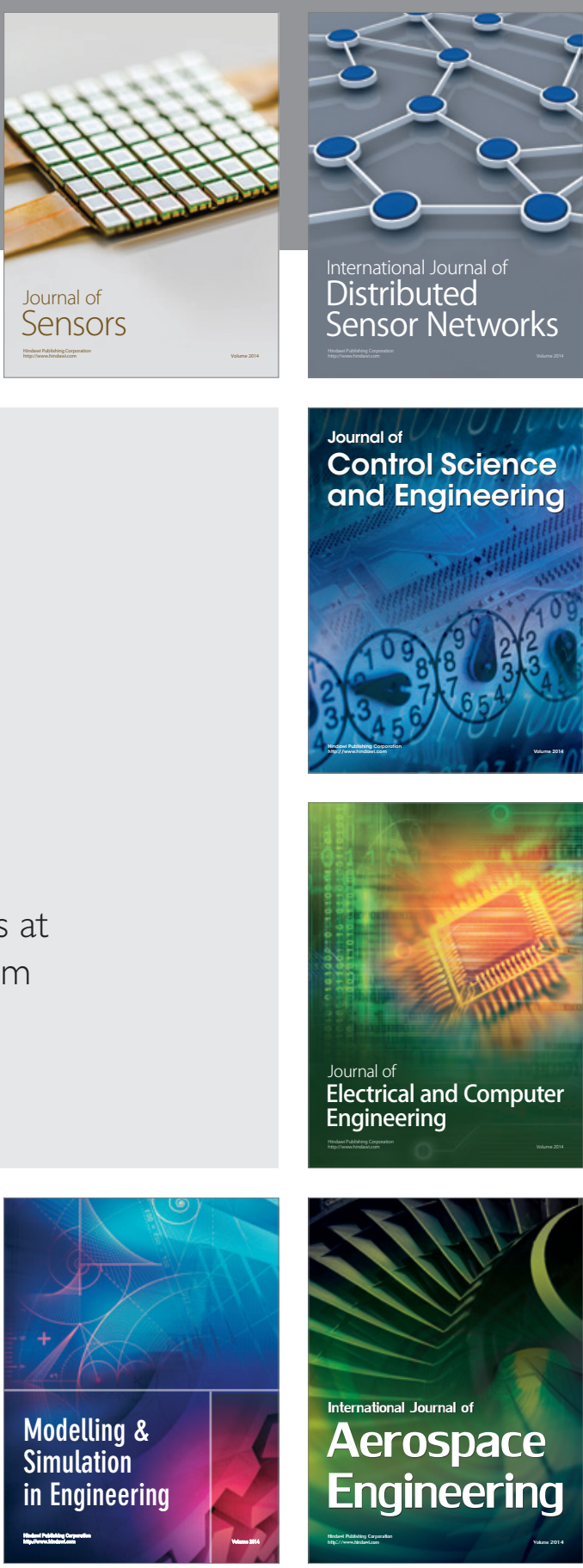

International Journal of

Distributed

Sensor Networks

$-$

Joumal of

Control Science

and Engineering
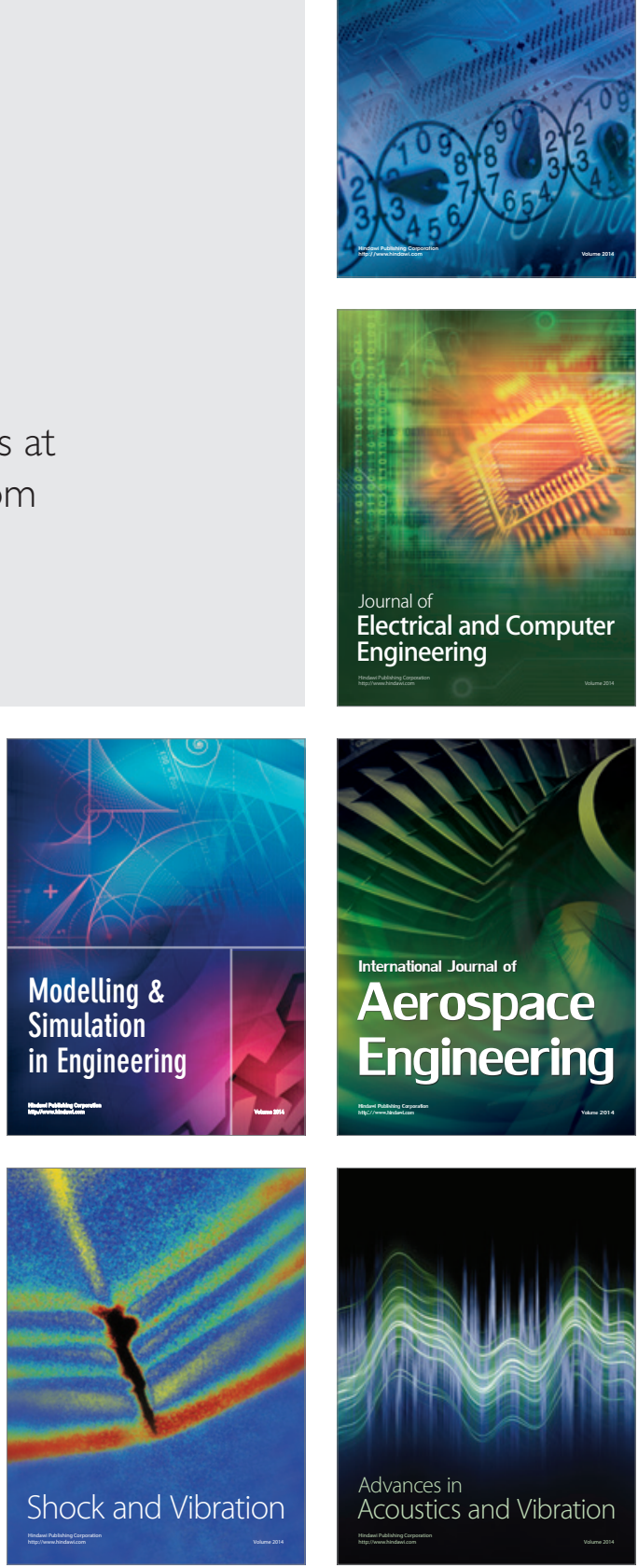